\title{
SCIENTIFIC NOTE
}

\section{Parasitismo de Pupas de Argon lota Hewitson (Lepidoptera: Hesperiidae) por Brachymeria pandora (Crawford) (Hymenoptera: Chalcididae) no Rio Grande do Sul}

\author{
Geraldo Salgado-Neto ${ }^{1}$, Rocco A Di Mare ${ }^{1}$, Marcelo Lopes-Da-Silva ${ }^{2}$ \\ ${ }^{1}$ Depto de Biologia, Centro de Ciências Naturais e Exatas - CCNE, Univ Federal de Santa Maria, Campus \\ Universitário, Faixa de Camobi, km 9, 97.119-900, Santa Maria, RS, Brasil; gsalgado@ bol.com.br; ${ }^{2}$ Unidade \\ Laboratorial de Entomologia, Lab de Quarentena Vegetal, Embrapa Recursos Genéticos e Biotecnologia, \\ Av. W5 Norte (final), CP 02372, 70770-917, Brasília, DF, Brasil; msilva@cenargen.embrapa.br \\ Edited by Madelaine Venzon - EPAMIG \\ Neotropical Entomology 39(2):311-312 (2010)
}

Parasitism of Argon lota Hewitson (Lepidoptera: Hesperiidae) by Brachymeria pandora (Crawford) (Hymenoptera: Chalcididae) in Rio Grande do Sul, Brazil

\begin{abstract}
This note reports the first record of parasitism of Argon lota Hewitson pupae by the facultative gregarious parasitoid Brachymeria pandora (Crawford). Up to six adults (three males and three females) of $B$. pandora were observed to emerge from a single $A$. lota pupa.
\end{abstract}

KEY WORDS: Natural enemy, biological control, palm tree pest, parasitoid wasp

Argon lota Hewitson apresenta ampla distribuição geográfica na região neotropical: México (SalinasGutiérres et al 2005), Argentina (Misiones, Corrientes, Entre Rios, Buenos Aires), Panamá, Colômbia, Trinidad, Brasil (Lamas 2004) e Costa Rica (Janzen \& Hallwachs 2009). No Brasil, está presente no Rio Grande do Sul (Biezanko 1963, Mielke 2005) e no Distrito Federal (Pinheiro \& Emery 2006). São citadas como plantas hospedeiras palmeiras da família Arecaceae: na Argentina os gêneros Acrocomia e Bactris por Canals (2003) e na Costa Rica as espécies Acrocomia aculeata, Astrocaryum alatum, Bactris guineensis e Chrysolidocarpus lutescens (Janzen \& Hallwachs 2009).

Foram coletadas dez pupas de A. lota entre março de 2006 e março de 2007, com varredura em folhas de 38 plantas de Syagrus romanzoffianum (Palmaceae), situadas no Campus da Universidade Federal de Santa Maria (UFSM), RS ( $29^{\circ} 42^{\prime}$ S e $\left.53^{\circ} 48^{\prime} \mathrm{W}\right)$. As pupas foram individualizadas e mantidas em câmara climatizada $\left(25 \pm 1^{\circ} \mathrm{C}\right.$; 70 \% UR; fotofase de 14h) e observadas diariamente até a emergência de adultos de $A$. lota ou parasitóides. Foram observados 28 espécimes de parasitóides emergidos das pupas coletadas, sendo registrado um máximo de seis parasitóides (três machos e três fêmeas) e um mínimo de um parasitóide por pupa. A proporção sexual foi 0,50 (machos/total). Os adultos foram fixados em etanol 70\%. Os espécimes voucher foram depositados na coleção do Laboratório de Biologia Evolutiva da Universidade Federal de Santa Maria. Posteriormente, os parasitóides foram enviados para especialistas e identificados como Brachymeria pandora (Crawford).

A ocorrência de $B$. pandora é registrada na América do
Sul, sendo encontrada na Guiana Inglesa (Crawford 1914) como parasitóide de larvas de espécies indeterminadas de Hesperiidae, e, na Venezuela, como parasitóide de pupas de Calpodes ethlius (Stoll) (Lepidoptera: Hesperiidae) (Teran 1980). Espécies próximas de B. pandora, tais como B. mnestor (Walker) e B. annulata (Fabricius), também são parasitóides gregários de pupas de algumas famílias de lepidópteros como Noctuidae, Nymphalidae, Papilionidae, Pieridae e Pyralidae (Gil-Santana \& Tavares 2005). No Brasil, B. pandora foi encontrada parasitando pupas de Historis odius (F.) (Lepidoptera: Nymphalidae) em Goiás, Espírito Santo e Rio de Janeiro (Marchiori et al 2003, GilSantana \& Tavares 2005). Esta nota é o primeiro registro de pupas de $A$. lota como hospedeiro de B. pandora e a primeira constatação desse parasitóide no Rio Grande do Sul.

\section{Agradecimentos}

Aos Drs Gerard Delvare (CIRAD, Montpellier, França) e Marcelo T Tavares (Universidade Federal do Espírito Santo) pela identificação do parasitóide. À Dra Mirna M Casagrande e ao Dr Olaf H H Mielke, (Universidade Federal do Paraná) pela identificação do lepidóptero e aos revisores anônimos da revista pelas sugestões e questionamentos realizados.

\section{Referências}

Biezanko C M (1963) Hesperiidae da Zona Sueste do Rio Grande do Sul (Contribuição ao conhecimento da fisiografia do Rio 
Grande do Sul). Arquivos de Entomologia. Escola de Agronomia "Eliseu Maciel” (A) 6: 1-24.

Canals G R (2003) Mariposas de Misiones. Buenos Aires, L.O.L.A., 492p.

Crawford J C (1914) New Parasitc Hymenoptera from British Guiana. Proc Entomol Soc Wash 16: 87-88.

Gil-Santana H R, Tavares M T (2005) Brachymeria pandora (Crawford) (Hymenoptera, Chalcididae): a new parasitoid of Historis odius (Fabricius) (Lepidoptera, Nymphalidae). Rev Bras Zool 22: 1211-1212.

Janzen D H, Hallwachs W (2009) Philosophy, navigation and use of a dynamic database (“ACG caterpillars SRNP”) for an inventory of the macro-caterpillar fauna, and its food plants and parasitoids, of the area de conservation Guanacaste (ACG), Northwestern Costa Rica. (http://janzen.sas.upenn.edu), acesso em 8.5.2009.

Lamas G (2004) Checklist: Part 4A. Hesperioidea - Papilionoidea. In Heppner J B (eds) Atlas of Neotropical Lepidoptera 5A. Gainesville, Association for Tropical Lepidoptera, 439p.

Marchiori C H, Penteado-Dias A M, Tavares M T (2003) Parasitoids of the family Chalcididae collected in pastures and forests using yellow traps, in Itumbiara, Goiás, Brasil. Braz J Biol 63: 357- 360 .

Mielke, O H (2005) Catalogue of the American Hesperioidea: Hesperiidae (Lepidoptera). Volume 1. Complementary and supplementary parts to the checklist of the Neotropical region. Hesperioidea: Hesperiidae: Pyrrhopyginae. Curitiba, Soc Bras Zool, 125p.

Pinheiro C E G, Emery E O (2006) As borboletas (Lepidoptera: Papilionoidea e Hesperioidea) da Área de Proteção Ambiental do Gama e Cabeça de Veado, Distrito Federal, Brasil. Biota Neotrop 6: 1-3

Salinas-Gutiérres J L, Warren, A D, Luis-Martinez A (2005) Hesperioidea (Lepidoptera: Rhopalocera) del occidente de México. Folia Entomol Mex 44: 305-320.

Teran B J (1980) Lista preliminar de Hymenoptera parasitos de otros insectos em Venezuela. Rev Fac Agron 11: 283-389.

Received 17/IX/08. Accepted 11/VIII/09. 